\title{
Quantum criticality in a generalized Dicke model
}

\author{
Yong $\mathrm{Li}^{1,2}$ Z. D. Wang, ${ }^{2}$ and C. P. Sun ${ }^{1}$ \\ ${ }^{1}$ Institute of Theoretical Physics \& Interdisciplinary Center of Theoretical Studies, Chinese Academy of Sciences, \\ Beijing 100080, China \\ ${ }^{2}$ Department of Physics \& Center of Theoretical and Computational Physics, The University of Hong Kong, \\ Pokfulam Road, Hong Kong, China
}

(Received 13 April 2006; published 23 August 2006)

\begin{abstract}
We employ a generalized Dicke model to study theoretically the quantum criticality of an extended two-level atomic ensemble interacting with a single-mode quantized light field. Effective Hamiltonians are derived and diagonalized to investigate numerically their eigenfrequencies for different quantum phases in the system. Based on the analysis of the eigenfrequencies, an intriguing quantum-phase transition from a normal phase to a superradiant phase is revealed clearly, which is quite different from that observed with a standard Dicke model.
\end{abstract}

DOI: 10.1103/PhysRevA.74.023815

PACS number(s): 42.50.Fx, 05.70.Jk, 73.43.Nq

\section{INTRODUCTION}

Quantum-phase transition (QPT) and quantum-critical phenomena, which are induced by the change of parameters and are accompanied by a dramatic change of physical properties, occur at zero temperature in many-body quantum systems [1]. Usually, a QPT may emerge in the parameter region where there is the energy-level crossing or the symmetry-breaking. QPTs have been mainly studied in connection with correlated electron and spin systems in condensed matter physics [1]. Very recently, it has also been paid much attention in the light-atoms interacting systems $[2,3]$, which enables us to understand the transition from radiation to superradiation from a different view point.

The systems of atomic ensembles interacting with optical fields have been studied both experimentally and theoretically, e.g., the electromagnetic-induced transparency [4] and the quantum storage of photon states $[5,6]$. The thermal phase-transition phenomena [7] have been studied in the Dicke model [8] (that is, a two-level atomic ensemble coupling with optical field) or generalized Dicke models [9]. In particular, the QPT in a radiation-matter interacting system was recently explored based on the Dicke model, but merely with the single-mode Dicke model $[2,3,10,11]$. When the coupling parameter $\lambda$ varies from that less than the critical value $\lambda_{c}$ to that larger than $\lambda_{c}$, the system goes from the normal phase to the superradiant one in the presence of the symmetry breaking. As the precursors of the QPT, the onset of chaos [2] and the entanglement properties [10] were studied in detail. However, it is noticed that these studies focused only on atomic ensembles with small dimensions compared with the optical wavelength, in which the dipole approximation can be used $[2,3,10]$. In this special case, the light-atoms interaction is irrelevant to the spatial positions of atoms. But, generally speaking, a realistic atomic ensemble may extend in a large scale so that light-atoms interaction is spatially dependent $[8,12]$.

In this paper, an exotic QPT phenomenon is investigated theoretically by developing the Dicke model for a more general case beyond the dipole approximation. We find that a kind of quantum-critical phenomenon also occurs in this ex- tended atomic ensemble, in which each atom interacts with a single-mode quantized light field; but the quantum criticality is quite different from that deduced from the spatially independent Dicke model [2]. In the present study, a normal phase and four possible superradiant phases are found, with only one of the four exhibiting the same critical point as that in the normal phase. Remarkably, it is shown that groundstate energy in the above-mentioned superradiant phase, connects continuously to the normal phase one at the critical point, but its second derivative does not.

\section{A GENERALIZED DICKE MODEL FOR AN EXTENDED ATOMIC ENSEMBLE}

Let us consider an extended ensemble with $N$ identical two-level atoms interacting with a single-mode quantized light field. Here, the spatial dimension of the atomic ensemble is much larger than the optical wavelength of the field. This radiation-matter system is usually described by a generalized Dicke model [8] with the Hamiltonian $H=H_{0}$ $+H_{I}$,

$$
\begin{gathered}
H_{0}=\omega a^{\dagger} a+\omega_{0} \sum_{j=1}^{N} \sigma_{\mathrm{ee}}^{(j)}, \\
H_{I}=\frac{\lambda}{\sqrt{N}} \sum_{j=1}^{N}\left(a^{\dagger} e^{-i k r_{j}}+a e^{i k r_{j}}\right)\left(\sigma_{\mathrm{eg}}^{(j)}+\sigma_{\mathrm{ge}}^{(j)}\right) .
\end{gathered}
$$

Here, $\hbar=1, k$ is the wave vector of the quantized light field, and $r_{j}$ is the position of the $j$ th atom; $\sigma_{\text {ee }}^{(j)}$ is the population operator of the $j$ atom; $\sigma_{\mathrm{eg}}^{(j)}$ is the flip operator between the excited state $|e\rangle$ and ground state $|g\rangle$ of the $j$ atom with the same energy differences $\omega_{0}$ for all the atoms; $a\left(a^{\dagger}\right)$ is the annihilation (creation) operator of the quantized light field, with $\lambda$ the relative coupling parameter. For simplicity, the ensemble is assumed to be one-dimensional with its direction along the wave vector.

Different from a standard Dicke model for smalldimension atomic ensembles [2], the spatial-dependent factors $\exp \left( \pm i k r_{j}\right)$ are taken into account seriously though the 
momentum of the center of mass can be neglected. It is also remarked that the terms connected to the nonrotatingwave scenario are still kept in Hamiltonian (1); in fact, if the rotating-wave approximation (RWA) were used, the factors $\exp \left( \pm i k r_{j}\right)$ would be absorbed into $\sigma_{\mathrm{eg}}^{(j)}$ and $\sigma_{\mathrm{ge}}^{(j)}[13,14]$.

\section{NORMAL PHASE} [6]:

We can first introduce the following collective operators

$$
B^{\dagger}=\frac{1}{\sqrt{N}} \sum_{j=1}^{N} \sigma_{\mathrm{eg}}^{(j)} e^{i k r_{j}}, \quad C^{\dagger}=\frac{1}{\sqrt{N}} \sum_{j=1}^{N} \sigma_{\mathrm{eg}}^{(j)} e^{-i k r_{j}} .
$$

It is obvious that in the limit of large $N$ with a small number of excitations (referred to as the normal phase), namely, the excitation numbers in states $|e\rangle$ are much less than $N$, the above two operators approximately satisfy the independent bosonic commutation relations

$$
\left[B, B^{\dagger}\right] \approx\left[C, C^{\dagger}\right] \approx 1, \quad\left[B, C^{\dagger}\right] \approx 0
$$

in the present extended ensemble, and can approximately be reexpressed as the two independent Bose operators $b^{\dagger}$ and $c^{\dagger}$,

$$
\left[b, b^{\dagger}\right]=\left[c, c^{\dagger}\right]=1, \quad\left[b, c^{\dagger}\right]=0 .
$$

In the present normal phase case, the original radiationmatter system described by Eq. (1) is approximated as a coupling three-mode bosonic system with the "low energy" effective Hamiltonian

$$
H^{(n)}=\omega_{0}\left(b^{\dagger} b+c^{\dagger} c\right)+\omega a^{\dagger} a+\lambda a^{\dagger}\left(c^{\dagger}+b\right)+\text { H.c. }
$$

We now apply a Bogoliubov transformation to diagonalize the above quadratic Hamiltonian (5) [15]. First, we rewrite it as

$$
H^{(n)}=\frac{1}{2} \mathrm{U}^{(n) \dagger} M^{(n)} \mathrm{U}^{(n)}-\frac{1}{2} \operatorname{tr} A^{(n)},
$$

where the operator-valued vectors $\mathrm{U}^{(n)}$ and the matrices $M^{(n)}$, $A^{(n)}, B^{(n)}$ are defined as

$$
\begin{gathered}
\mathrm{U}^{(n)}=\left(a, b, c ; a^{\dagger}, b^{\dagger}, c^{\dagger}\right)^{T}, \\
M^{(n)}=\left(\begin{array}{cc}
A^{(n)} & B^{(n)} \\
B^{(n)^{*}} & A^{(n)^{*}}
\end{array}\right), \quad A^{(n)}=\left(\begin{array}{ccc}
\omega & \lambda & 0 \\
\lambda & \omega_{0} & 0 \\
0 & 0 & \omega_{0}
\end{array}\right), \\
B^{(n)}=\left(\begin{array}{ccc}
0 & 0 & \lambda \\
0 & 0 & 0 \\
\lambda & 0 & 0
\end{array}\right) .
\end{gathered}
$$

According to Ref. [15], we diagonalize the Hamiltonian (6) in two steps: (i) find a unitary canonical transformation $T^{(n)}$ such that $T^{(n)} \hat{\eta} T^{(n) \dagger} \hat{\eta}=1, T^{(n)^{*}}=\hat{\gamma} T^{(n)} \hat{\gamma}$, where

$$
\hat{\eta}=\left(\begin{array}{cc}
1 & 0 \\
0 & -1
\end{array}\right), \quad \hat{\gamma}=\left(\begin{array}{ll}
0 & 1 \\
1 & 0
\end{array}\right),
$$

and (ii) introduce the quasiparticle operators

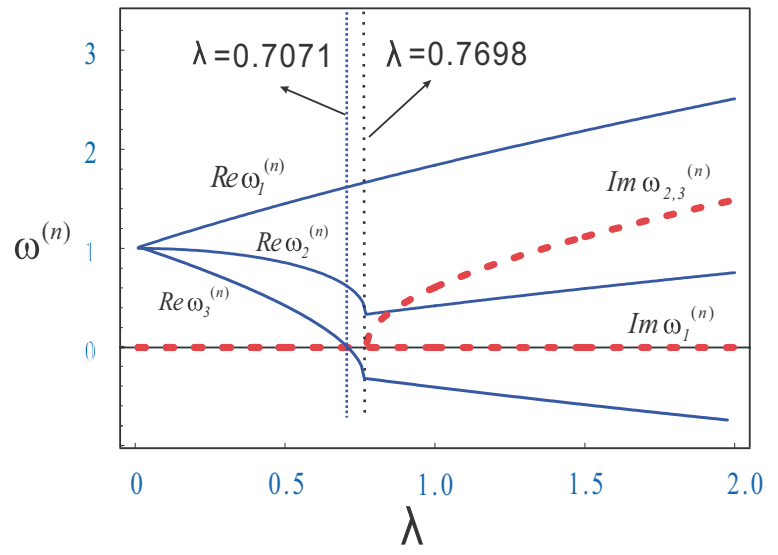

FIG. 1. (Color online) The real part (thin solid lines) and imaginary part (thick dashed lines) of the eigenfrequencies vs the coupling parameter $\lambda$ in units of $\omega_{0}$ in the normal phase at the resonance case $\omega=\omega_{0}$.

$$
\mathrm{V}^{(n)}=T^{(n)} \mathrm{U}^{(n)}=\left(h_{1}, h_{2}, h_{3} ; h_{1}^{\dagger}, h_{2}^{\dagger}, h_{3}^{\dagger}\right)^{T} .
$$

Then the Hamiltonian (6) is cast into a diagonalized form

$$
H^{(n)}=\sum_{i=1}^{3} \omega_{i}^{(n)}\left(h_{i}^{\dagger} h_{i}+\frac{1}{2}\right)-\frac{1}{2} \operatorname{tr} A^{(n)}
$$

and describes the quasiparticle excitations with frequencies $\omega_{1,2,3}^{(n)}$, which are obtained by diagonalizing $\hat{\eta} M^{(n)}$ with $T^{(n)}$ into

$$
T \hat{\eta} M T^{-1}=\Omega=\left(\begin{array}{cc}
\omega & 0 \\
0 & -\omega
\end{array}\right), \quad \omega=\left(\begin{array}{ccc}
\omega_{1} & & \\
& \omega_{2} & \\
& & \omega_{3}
\end{array}\right) .
$$

Below, we focus only on the properties of eigenfrequencies in order to explore the existence of quantum criticality. Since the matrix $M^{(n)}$ is of $6 \times 6$, general analytic results for the diagonalization of $\hat{\eta} M^{(n)}$ are difficult to obtain. Nevertheless, we can diagonalize it numerically to obtain the eigenfrequencies $\omega_{1,2,3}^{(n)}[16]$. The related canonical transformation matrix $T^{(n)}$ can also be obtained numerically. Figure 1 shows the numerical results for the real and imaginary parts of $\omega_{1,2,3}^{(n)}$. For simplicity, we illustrate the resonant case $\omega=\omega_{0}=1$ here. Certainly, the nonresonance cases can also be studied numerically, with similar features being revealed. As seen from Fig. 1, when $\lambda>0.7698$, the imaginary parts of two eigenfrequencies are nonzero. This means that the corresponding eigenfrequencies are complex and thus the eigenstate is unstable and physically impossible. But it is inappropriate to consider naively that $\lambda=0.7698$ as the critical point. Since the eigenvalue $\omega_{3}^{(n)}$ is negative in the range of $\lambda$ $\in(0.7071,0.7698)$, a negative eigenfrequency of the boson mode is not allowed physically either. Therefore, in the resonance case $\omega=\omega_{0}(=1)$, a real critical point is located at $\lambda_{c}^{(n)}=0.7071=\sqrt{2} / 2$. In addition, for a general case, the critical point is found to be $\lambda_{c}^{(n)}=\sqrt{\omega \omega_{0} / 2}$. 


\section{SUPERRADIANT PHASE}

In order to describe excitations in the parameter region above the critical point, we now incorporate the fact that both the field and the atomic collective excitations acquire macroscopic occupations, namely, the above approximation to neglect the number of excitations over $N$ is no longer valid [2]. To this aspect, the introduced collective operators $B^{\dagger}$ and $C^{\dagger}$ in Eq. (2) should be expressed approximately as [17]

$$
B^{\dagger}=b^{\dagger} \sqrt{1-\frac{b^{\dagger} b}{N}}, \quad C^{\dagger}=c^{\dagger} \sqrt{1-\frac{c^{\dagger} c}{N}},
$$

in terms of the Bose operators $b^{\dagger}, b, c^{\dagger}$, and $c$. This transformation maps the original light-atoms system to a coupling three-mode bosonic system with the Hamiltonian

$$
\begin{aligned}
H^{(s)}= & \omega_{0}\left(b^{\dagger} b+c^{\dagger} c\right)+\omega a^{\dagger} a+\lambda a^{\dagger} \\
& \times\left(c^{\dagger} \sqrt{1-\frac{c^{\dagger} c}{N}}+\sqrt{1-\frac{b^{\dagger} b}{N}} b\right)+\text { H.c. }
\end{aligned}
$$

For the present superradiant phase, the bosonic modes may be displaced in the following way:

$$
a^{\dagger} \rightarrow d^{\dagger}+\alpha^{*}, \quad b^{\dagger} \rightarrow e^{\dagger}-\beta^{*}, \quad c^{\dagger} \rightarrow f^{\dagger}-\gamma^{*},
$$

where $\alpha, \beta$, and $\gamma$ are generally complex parameters in the order of $O(\sqrt{N})$ [2] to be determined later. This is equivalent to assuming that all modes behave as the nonzero, macroscopic mean fields above $\lambda_{c}^{(n)}$.

Keeping the terms up to the order of $O\left(N^{0}\right)$, the Hamiltonian (12) becomes

$$
\begin{aligned}
H^{(s)}= & \left\{\lambda \sqrt{\frac{k_{f}}{N}}\left[d^{\dagger} f^{\dagger}+\frac{\left(\alpha^{*} f^{\dagger}-\gamma^{*} d^{\dagger}\right)}{2 k_{f}}\left(\gamma f^{\dagger}+\gamma^{*} f\right)\right]\right. \\
& \left.+\lambda \sqrt{\frac{k_{e}}{N}}\left[d e^{\dagger}+\frac{\left(\alpha e^{\dagger}-\beta^{*} d\right)}{2 k_{e}}\left(\beta e^{\dagger}+\beta^{*} e\right)\right]+\text { H.c. }\right\} \\
& +\omega d^{\dagger} d+\omega_{e} e^{\dagger} e+\omega_{f} f^{\dagger} f+\mathrm{c}_{0}^{(s)},
\end{aligned}
$$

where the constant term

$$
\mathrm{c}_{0}^{(s)}=\omega|\alpha|^{2}+\omega_{0}\left(|\beta|^{2}+|\gamma|^{2}\right)-\frac{2 \lambda \alpha}{\sqrt{N}}\left(\beta^{*} \sqrt{k_{e}}+\gamma \sqrt{k_{f}}\right)
$$

will substantially contribute to the ground-state energy at a critical point; the renormalized frequencies

$$
\omega_{e}=\omega_{0}+\lambda \alpha^{*} \beta / \sqrt{N k_{e}}, \quad \omega_{f}=\omega_{0}+\lambda \alpha \gamma / \sqrt{N k_{e}},
$$

with $k_{e}=N-|\beta|^{2}$ and $k_{f}=N-|\gamma|^{2}$. In the derivation of Eq. (14), the terms being linear in the bosonic operators are eliminated by choosing the appropriate displacements $\alpha, \beta$, and $\gamma$ in the following four cases:

$$
\begin{aligned}
& \alpha^{(1,2,3,4)}=\frac{2 e^{i \phi} \lambda}{\omega} \sqrt{\frac{X_{+} X_{-}}{N},} \\
& \beta^{(j)}=e^{i \phi} \sqrt{X_{\mp}} \quad(j=1,3), \\
& \gamma^{(j)}=e^{-i \phi} \sqrt{X_{\mp}} \quad(j=1,3),
\end{aligned}
$$

$$
\begin{aligned}
& \beta^{(j)}=e^{i \phi} \sqrt{X_{ \pm}} \quad(j=2,4), \\
& \gamma^{(j)}=e^{-i \phi} \sqrt{X_{\overline{+}}} \quad(j=2,4),
\end{aligned}
$$

where $X_{ \pm}=\frac{N}{2}\left(1 \pm \frac{\omega \omega_{0}}{2 \lambda^{2}}\right)$, and $\phi$ is an arbitrary real number relating to the phases of displacements. In fact, we see from the form of $\alpha^{(j)}$ in Eq. (16) that only when

$$
4 \lambda^{4}-\omega^{2} \omega_{0}^{2} \geqslant 0,
$$

$\alpha^{(j)}$ can be physically meaningful. Thus in the following discussions, it is required that

$$
\lambda \geqslant \sqrt{\frac{\omega \omega_{0}}{2}}\left(=\lambda_{c}^{(n)}\right)
$$

It is interesting to note that this threshold is just the critical point determined in the normal phase case.

Since $H^{(s)}(\phi)$ in Eq. (14) can be transferred to a $\phi$-independent Hamiltonian $H^{(s)}(\phi \equiv 0)$ through a unitary transformation

$$
U(\phi)=e^{i \phi\left(d^{\dagger} d+e^{\dagger} e-f^{\dagger} f\right),}
$$

we need only to look into the spectra of $H^{(s)}(0)$ in the four cases specified by Eq. (16), respectively. Because $H^{(s)}(0)$ is quadratic in each case, which is diagonalized by using the same method presented above as

$$
H^{(j)}=\sum_{i=1}^{3} \omega_{i}^{(j)}\left(e_{i}^{(j) \dagger} e_{i}^{(j)}+\frac{1}{2}\right)-\frac{1}{2} \operatorname{tr} A^{(j)}+\mathrm{c}_{0}^{(j)},
$$

for the four cases $j=1, \ldots, 4$. Here, the quasiparticle excitation is described by the boson-vector operators

$$
\begin{aligned}
e^{(j)} & =\left(e_{1}^{(j)}, e_{2}^{(j)}, e_{3}^{(j)} ; e_{1}^{(j) \dagger}, e_{2}^{(j) \dagger}, e_{3}^{(j) \dagger}\right)^{T} \\
& =T^{(j)}\left(d^{(j)}, e^{(j)}, f^{(j)} ; d^{(j) \dagger}, e^{(j) \dagger}, f^{(j) \dagger}\right)^{T}
\end{aligned}
$$

in the $j$ th phase, where

$$
d^{(j)}=a-\left|\alpha^{(j)}\right|, \quad e^{(j)}=b+\left|\beta^{(j)}\right|, \quad f^{(j)}=c+\left|\gamma^{(j)}\right|
$$

according to Eq. (13). $T^{(j)}$ is still the introduced unitary transformation to diagonalize

$$
\hat{\eta} M^{(j)}=\left(\begin{array}{cc}
A^{(j)} & B^{(j)} \\
-B^{(j)} & -A^{(j)}
\end{array}\right),
$$

where

$$
\begin{array}{cc}
A^{(1,3)}=\left(\begin{array}{ccc}
\omega & A_{\mp} & C_{\mp} \\
A_{\mp} & \omega_{\bar{\mp}} & 0 \\
C_{\bar{\mp}} & 0 & \omega_{\mp}
\end{array}\right), & B^{(1,3)}=\left(\begin{array}{ccc}
0 & C_{\mp} & A_{\mp} \\
C_{\mp} & B_{\mp} & 0 \\
A_{\mp} & 0 & B_{\mp}
\end{array}\right), \\
A^{(2,4)}=\left(\begin{array}{ccc}
\omega & A_{ \pm} & C_{\mp} \\
A_{ \pm} & \omega_{ \pm} & 0 \\
C_{\mp} & 0 & \omega_{\mp}
\end{array}\right), & B^{(2,4)}=\left(\begin{array}{ccc}
0 & C_{ \pm} & A_{\mp} \\
C_{ \pm} & B_{ \pm} & 0 \\
A_{\mp} & 0 & B_{\mp}
\end{array}\right),
\end{array}
$$

with

$$
\omega_{ \pm}:=\omega_{0}+4 \lambda^{2} X_{ \pm} / N \omega, \quad B_{ \pm}:=2 \lambda^{2} X_{ \pm} / N \omega
$$




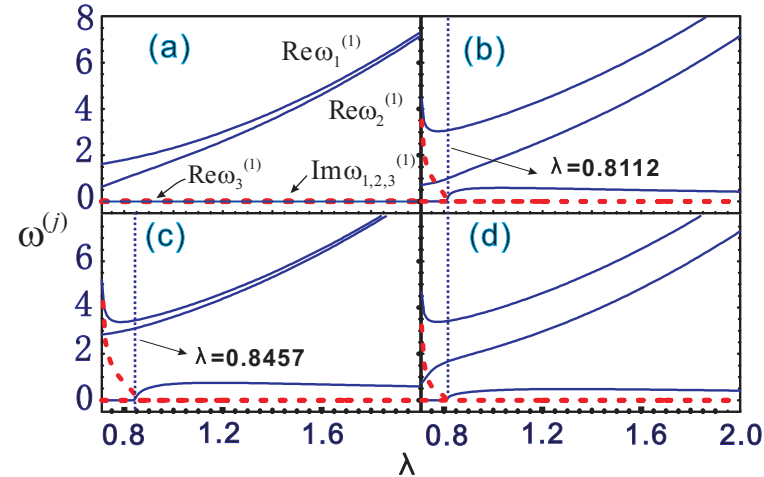

FIG. 2. (Color online) The real part (solid lines) and imaginary part (dashed lines) of the eigenfrequencies vs the coupling parameter $\lambda$ in units of $\omega_{0}$ in the superradiant phase at the resonance case $\omega=\omega_{0}$, where (a), (b), (c), (d) correspond respectively to the cases (1), (2), (3), (4) specified in the text.

$$
A_{ \pm}:=\lambda\left(X_{\mp}-X_{ \pm} / 2\right) / \sqrt{N X_{\mp}}, \quad C_{ \pm}:=-\lambda X_{ \pm} / 2 \sqrt{N X_{\mp}} .
$$

Clearly, $\omega_{i}^{(j)}(i=1,2,3)$ is the $i$ th eigenfrequency for the Hamiltonian $H^{(j)}$. Note that the canonical transformation matrix $T^{(j)}$ can be obtained numerically in the numerical diagonalization of $\hat{\eta} M^{(j)}$.

The numerical results of eigenfrequencies $\omega_{1,2,3}^{(j)}$ vs the coupling parameter $\lambda$ are plotted in Fig. 2. The curves for both the real and imaginary parts of eigenfrequencies of $H^{(1)}$ in the resonant case are shown in Fig. 2(a). It is found that the eigenfrequencies are physically reasonable when $\lambda$ $>0.7071$ since the imaginary parts of all the eigenfrequencies are zero. This means a "quantum phase" emerges above the critical point

$$
\lambda_{c}^{(1)}=\sqrt{\frac{\omega \omega_{0}}{2}}=0.7071 .
$$

It is seen from Fig. 2(a) that the eigenfrequency $\omega_{3}^{(1)}$ is always zero above $\lambda_{c}^{(1)}$, which implies that $H^{(1)}$ is reduced to have two independent boson modes. It is remarkable that the critical point is just the same one as that determined in the normal phase $\lambda_{c}^{(1)}=\lambda_{c}^{(n)}=\lambda_{c}$, demonstrating the consistency of our analysis.

In Fig. 2(b) [Fig. 2(d)], the numerical results for the real and imaginary parts of three eigenfrequencies of $H^{(2)}\left(H^{(4)}\right)$ in the resonant case are shown. As seen from Fig. 2(b) [Fig. 2(d)], only when

$$
\lambda>\lambda_{c}^{(2)}=0.8112 \quad\left(\lambda_{c}^{(4)}=0.8112\right),
$$

another possible "quantum phase" may appear as the imaginary parts of all eigenfrequencies are zero. While for $H^{(3)}$, as seen from Fig. 2(c), only when $\lambda>0.8457=\lambda_{c}^{(3)}$, the imaginary part of all the eigenfrequencies are zero, indicating a possible "quantum phase."

In Fig. 3(a), we plot together the eigenfrequencies vs $\lambda$ for the normal phase and first superradiant phase. The eigenfrequencies $\omega_{i}^{(n)}$ and $\omega_{i}^{(1)}$ (for $i=1,2,3$ ) are continuous at the critical point, respectively. Comparing with the results in the spatially independent Dicke model [2], our numerical studies
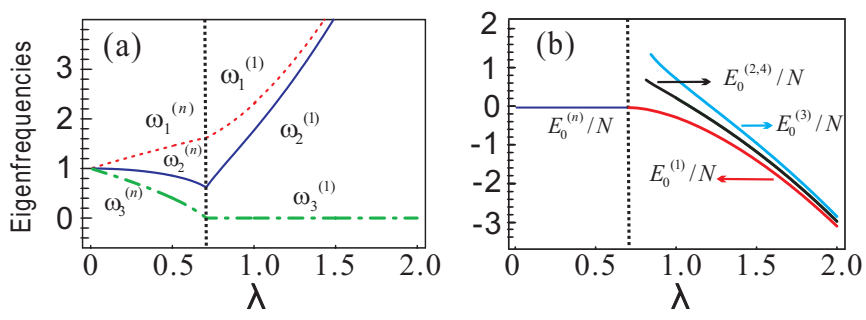

FIG. 3. (Color online) (a) The eigenfrequencies for normal phase and the first superradiant phase. (b) The ground-state energy densities $\left(N=10^{6}\right)$ for the normal phase and the superradiant phases at the resonance case $\omega=\omega_{0}=1$.

show clearly that the excitation energy $\omega_{3}^{(n)}$ in the normal phase vanishes as $\left|\lambda-\lambda_{c}\right|^{z v}$ and the characteristic length scale $l_{3}=1 / \sqrt{\omega_{3}^{(n)}}$ diverges as $\left|\lambda-\lambda_{c}\right|^{-v}$ at the quantum transition point $\lambda_{c}$, with the exponents given by $v=1 / 2, z=2$ on resonance; however, it is interesting to note that no critical exponents for $\omega_{3}^{(1)}$ in the superradiant phase can be specified since $\omega_{3}^{(1)} \equiv 0$. Meanwhile, for the ground state, $\left\langle a^{\dagger} a\right\rangle_{G} / N=0$ below $\lambda_{c}$, while

$$
\frac{\left\langle a^{\dagger} a\right\rangle_{G}}{N}=\left|\alpha^{(1)}\right|^{2} / N \propto\left(\lambda-\lambda_{c}\right)
$$

above $\lambda_{c}$, i.e., the field is macroscopically occupied. So $\alpha^{(1)}$ may be understood as a kind of order parameter of the superradiant phase, whose critical exponent is $1 / 2$ above $\lambda_{c}$. In addition, Fig. 3(b) presents the ground-state energy densities as a function of coupling for all the possible phases. Clearly, the ground-state energy densities of the first superradiant phase is always the lowest one above $\lambda_{c}$, while the other three approach it in the large $\lambda$ limit; moreover, it connects continuously with that of the normal phase but possesses a discontinuity in its second derivative at $\lambda_{c}$ through a detailed numerical analysis. From this viewpoint, together with the fact that the same critical point is determined from both sides of the normal phase and the first one, it is most likely that only the first superradiant phase is a real physical one.

\section{REMARKS AND CONCLUSIONS}

Before concluding this paper, we wish to remark briefly on the origin of the QPT occurring in the present work. From Fig. 3(a), it is clear that the energy level of the first excited state of the system $\left(\omega_{3}^{(n)}+E_{0}^{(n)}\right)$ touches the ground-state energy level $E_{0}^{(n)}$ (or $E_{0}^{(1)}$ ) at the critical point. Obviously, it is this level touching that accounts for the emergence of the QPT and the corresponding quantum criticality in the present generalized Dicke model. It is also remarked that the $A^{2}$ terms (where $A$ is the vector potential) has been neglected here, as done in several previous works $[2,3,11]$, while the absence of $A^{2}$ terms $[18,19]$ seems to be crucial for the observed quantum-phase transition in the present model, namely, the presence of $A^{2}$ terms in the model Hamiltonian leads to vanishing of the criticality.

Although the effect of non-RWA terms may normally be negligibly small, the present work (also see Ref. [2]) illus- 
trates that it plays a meaningful role when the atomic number $N$ is large, e.g., the criticality differs from that with the RWA. On the other hand, for actual atoms that may not be pure two-level ones, other atomic transitions may occur and spoil the present model before the non-RWA terms become important. Nevertheless, the present study is still theoretically interesting and valuable, particularly relevant to some atomic systems (or artificial and effective atomiclike ones [20]) wherein the energy spacing of any other transitions is much larger than that of the considered two levels (or other transitions do not exist). For example, for a Dicke-like model consisting of many $1 / 2$ spins coupled to single mode bosonic field (by electrical dipole couplinglike type), other transitions do not exist in the spin systems. Then the counterrotating terms play an important role when the coupling parameter is close to the critical value.

In conclusion, based on a generalized Dicke model, we have investigated theoretically the quantum criticality of an extended atomic ensemble with a larger spatial dimension comparable to the optical wavelength of a quantized light field. A useful formalism is developed to study numerically eigenfrequencies of the system in different quantum phases. Comparing with the critical phenomenon around the critical point $\tilde{\lambda_{c}}=\sqrt{\omega \omega_{0}} / 2$ for atomic ensemble of small dimension [2], a rather different quantum criticality is revealed around the transition point $\left(\lambda_{c}=\sqrt{\omega \omega_{0} / 2}=\sqrt{2} \lambda_{c}\right)$ from the normal phase to the superradiant phase.

\section{ACKNOWLEDGMENTS}

This work was supported by the NSFC Grants No. 90203018, No. 10474104, No. 60433050, No. 10447133, No. 10574133, and No. 10429401, the NFRP of China Grants No. 2001CB309310 and No. 2005CB724508, the RGC grant of Hong Kong (Grant No. HKU7045/05P), and the URC fund of HKU.
[1] S. Sachdev, Quantum Phase Transitions (Cambridge University Press, Cambridge, 1999).

[2] C. Emary and T. Brandes, Phys. Rev. Lett. 90, 044101 (2003); Phys. Rev. E 67, 066203 (2003).

[3] J. Reslen, L. Quiroga, and N. F. Johnson, Europhys. Lett. 69, 8 (2005).

[4] S. E. Harris, Phys. Today 50, 36 (1997).

[5] M. D. Lukin, Rev. Mod. Phys. 75, 457 (2003).

[6] C. P. Sun, Y. Li, and X. F. Liu, Phys. Rev. Lett. 91, 147903 (2003).

[7] K. Rzazewski, K. Wódkiewicz, and W. Zakowicz, Phys. Rev. Lett. 35, 432 (1975).

[8] R. H. Dicke, Phys. Rev. 93, 99 (1954).

[9] F. T. Hioe, Phys. Rev. A 8, 1440 (1973).

[10] N. Lambert, C. Emary, and T. Brandes, Phys. Rev. Lett. 92, 073602 (2004).

[11] J. Vidal and S. Dusuel, Europhys. Lett. 74, 817 (2006).

[12] M. O. Scully, E. S. Fry, C. H. Raymond Ooi, and K. Wódkiewicz, Phys. Rev. Lett. 96, 010501 (2006).

[13] C. Mewes and M. Fleischhauer, Phys. Rev. A 72, 022327
(2005).

[14] Y. Li, L. Zheng, Yu-xi Liu, and C. P. Sun, Phys. Rev. A 73, 043805 (2006).

[15] Jean-Paul Blaizot, Quantum Theory of Finite Systems (Massachusetts Institute of Technology, Cambridge, 1986).

[16] Actually, for the particular $6 \times 6$ dimensional matrix $\hat{\eta} M^{(n)}$ in this normal phase, we have a simple method to diagonalize it by reducing it into two $3 \times 3$ dimensional submatrices (not presented here).

[17] C. P. Sun, S. X. Yu, and Y. B. Gao, e-print quant-ph/9809079; Yu-Xi Liu, C. P. Sun, S. X. Yu, and D. L. Zhou, Phys. Rev. A 63, 023802 (2001).

[18] K. Rzazewski and K. Wódkiewicz, Phys. Rev. A 43, 593 (1991).

[19] K. Rzazewski and K. Wódkiewicz, Phys. Rev. Lett. 96, 089301 (2006); V. Bužek, M. Orszag, and M. Roško, ibid. 96, 089302 (2006).

[20] F. Dimer, B. Estienne, A. S. Parkins, and H. J. Carmichael, e-print quant-ph/0607115. 